\title{
CARACTERIZACIÓN DE LA MATERIA PRIMA PARA LA ELABORACIÓN DE UN MATERIAL DE CONSTRUCCIÓN, UTILIZANDO DIATOMITA Y RESIDUOS DE CONSTRUCCIÓN Y DEMOLICIÓN (RCD)
}

\author{
J. R. SERRALDE, J. HERNÁNDEZ, E. CERECEDO, E. SALINAS, \\ M. I. REYES, V. RODRÍGUEZ, E. MORENO
}

Centro de Innovación y Desarrollo Tecnológico del Estado de Hidalgo Universidad Autónoma del Estado de Hidalgo, Ciudad del Conocimiento, Carretera Pachuca-Tulancingo km. 4.5, Mineral de la Reforma, Hidalgo, México

\section{RESUMEN}

Entre los Residuos de Construcción y Demolición (RCD) que se pueden reciclar, resaltan los agregados resultantes de la trituración. A pesar de que hay diversos estudios referentes a su uso en concreto, en la incorporación a bloques de construcción son limitados. Esta investigación tiene como objetivo elaborar bloques de pumicita a partir de la utilización de RCD y diatomita para mejorar sus propiedades mecánicas. Se llevaron a cabo diversas pruebas, primeramente, para determinar el porcentaje óptimo de diatomita en sustitución con el cemento y posteriormente la incorporación de los RCD en sustitución de la pumicita. Las pruebas han demostrado que el uso de diatomita y de RCD en la incorporación de bloques de pumicita podría dar una resistencia a la compresión mayor a la de los bloques que se comercializan actualmente. También se ha demostrado que se podría compensar el uso de una cantidad mayor de cemento al usar agregados reciclados en concretos.

Palabras clave: Reciclaje; Sustentable; Bloque; Puzolana; Diatomita 


\section{INTRODUCCIÓN}

El reciclaje ha adquirido mayor impacto, principalmente en el ámbito de la construcción ya que esta industria contiene bastantes elementos cuya gestión no es llevada a cabo de la forma correcta como los Residuos de Construcción y Demolición (RCD); y producen gran huella de carbono, entre ellos se encuentra el cemento, cuyo impacto ambiental genera un $7 \%$ de las emisiones totales de $\mathrm{CO}_{2}$ en el mundo (Oh et al., 2014). La sostenibilidad en la industria de la construcción es inevitable y no solo significa que se debe reducir la huella de carbono, sino que también debe contribuir a conservar los recursos naturales que son cruciales para el crecimiento continuo (Akhtar, 2018). Por lo que, al crear materiales de construcción que sean sustentables y con mejores características que los existentes, es una forma en la cual se puede reducir el impacto ambiental generado también por los Residuos de Construcción y Demolición.

Cada año se producen grandes cantidades de estos desechos, los cuales son considerados inertes, no peligrosos y poseen alta susceptibilidad de ser aprovechados mediante transformación y reincorporación como materia prima de agregados en la fabricación de nuevos productos. En su mayoría, los agregados de concreto reciclado están compuestos de una mezcla de pasta de mortero endurecida adherida. Una gran parte de ellos se podría reutilizar en la fabricación de nuevos materiales de construcción para disminuir las cantidades de desechos cuyo destino final son vertederos clandestinos como barrancas o terrenos cuyo uso se podría aprovechar para realizar actividades productivas y así preservar los recursos naturales (Serralde et al, 2015). El concepto de reciclaje de los RCD se ha estado abordando en diversos trabajos de investigación anteriores, sin embargo, son investigaciones a nivel local, por lo que en México aún no se tiene un control total de la disposición final de estos escombros.

Los bloques de pumicita son utilizados en la industria de la construcción como elementos perimetrales y divisorios en edificaciones, creando muros sólidos cuyas dimensiones son variables. Se consideran bloques de tepojal-cemento, donde su uso recae en un material no estructural en base a la normativa mexicana NMX-C-441-ONNCCE-2013. Una de las propiedades mecánicas más importantes de los bloques es la resistencia a la compresión. Las propiedades mecánicas pueden mejorarse con materiales alternativos utilizados para el reemplazo parcial del cemento Portland. Uno de estos materiales es la diatomita, que es una roca silícica de tipo fósil que se formó por la acumulación sedimentaria de capas silíceas de algas, compuesta de esqueletos opalinos de sílice amorfa y tiene una fórmula química de $\mathrm{SiO}_{2}-n \mathrm{H}_{2} \mathrm{O}$ (Mete $\mathrm{Z}, 1988$ ). Este tipo de $\mathrm{SiO}_{2}$ puede reaccionar con $\mathrm{Ca}(\mathrm{OH})_{2}$ y producir hidratos de silicato de calcio (CSH), que son responsables del desarrollo de la resistencia. Se caracteriza por ser una puzolana natural y ha sido utilizada en diversas investigaciones como aditivo puzolánico para cemento portland (Aydin y Gül, 2007). La reacción puzolánica de la diatomita conduce a la formación de mayores cantidades de CSH, especialmente a la edad de 28 días (Katis, 2006). El uso de rocas de diatomita como aditivos de cemento tiene inconvenientes tales como una mayor demanda de agua, pero la resistencia a la compresión de los cementos producidos en laboratorio exhibe valores más altos que los del cemento Portland de referencia (Stamatakis et al, 2003).

\section{MATERIALES Y MÉTODOS}

\subsection{Preparación de materias primas}

Las materias primas utilizadas en esta investigación fueron pumicita, cemento Portland compuesto (CPC), y diatomita. Tanto la pumicita como el cemento portland compuesto se obtuvieron de una casa de materiales (Pachuca de Soto / México). La diatomita se obtuvo de la región de Acatlán / Hidalgo (México) y se preparó en forma natural, calcinada y calcinada con fundente. En la preparación de las muestras de bloques de pumicita, se realizó en base a información obtenida de diversas bloqueras 
ubicadas en la región de Pachuca de Soto.

\subsection{Caracterización de las materias primas}

Las muestras de diatomita se molieron en un mortero y se realizó un tratamiento térmico (calcinación) a dos muestras de las muestras; una utilizando fundente de hidróxido de sodio $(\mathrm{NaOH})$ al $7 \%$; y la otra sin fundente. La calcinación de las muestras de diatomita se realizó utilizando un horno controlado por proceso Lindberg-ISB en atmósfera de aire (Figura 1). El proceso se programó de tal manera que creó un calentamiento de hasta $1000{ }^{\circ} \mathrm{C}$ a una velocidad de entre $10-20{ }^{\circ} \mathrm{C} / \mathrm{min}$ y una parada a $1000{ }^{\circ} \mathrm{C}$ durante 1 hora y luego se enfrió a temperatura ambiente esto con el fin de aumentar la cantidad de óxido de silicio, así como eliminar impurezas orgánicas y gases (Borgel 2007).

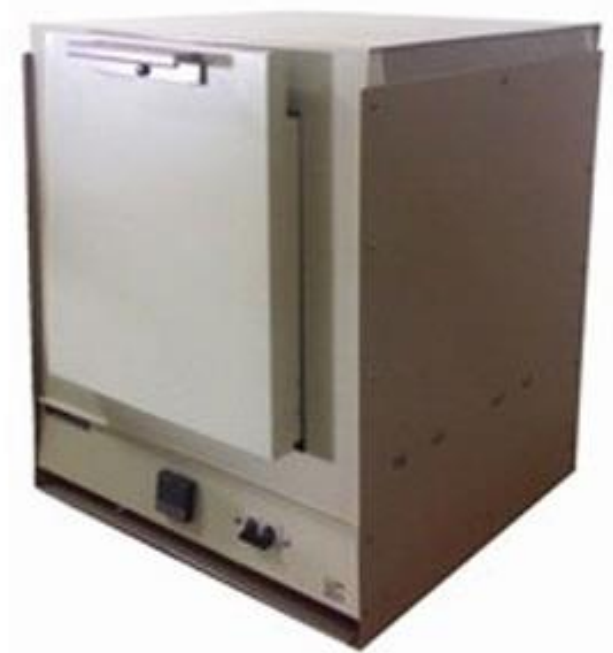

Figura 1. Horno Lindberg ISB ubicado en la Unidad Central de Laboratorios (UCL) en la Ciudad del Conocimiento, Universidad Autónoma del Estado de Hidalgo (UAEH). Disponible en http://www.rsulab.mx/product_info.php/products_id/287

Para obtener una muestra representativa de las materias primas, se les realizó la técnica de cuarteo. La composición mineralógica se determinó mediante difracción de rayos $\mathrm{X}$ (DRX), en un difractómetro Equinox 2000 (INEL, Artenay, Francia) con radiación CoK $\alpha 1$ (1.79026 ̊̊). Para esta técnica se tamizaron las muestras entre 74 y 53 micras $(\mu \mathrm{m})$ aproximadamente por las mallas 200 y 270 . Se colocaron en un porta-muestras de aluminio.

El análisis microestructural de las materias primas se realizó utilizando Microscopio Electrónico de Barrido con microanálisis de Energías Dispersivas de Rayos X JEOL modelo JSM-6300 (JEOL Ltd., Tokio, Japón). Se observó que las diatomitas naturales y calcinadas tenían formas similares, pero de diferentes tamaños; Las formas amorfas de las diatomeas y de los poros fueron dominantes en ambas diatomitas calcinadas.

\subsection{Elaboración de bloques de pumicita.}

La elaboración de los bloques se llevó a cabo en 2 etapas con el fin de determinar el que mejor propiedadesmecánicas presente. Primero se realizó la sustitución de cemento con diatomita en porcentajes de 5, 10 y $15 \%$. En la segunda etapa se incorporaron los agregados reciclados en el bloque de pumicita en porcentajes de 10,20 y $30 \%$.

Los bloques utilizados en las primeras pruebas se agruparon como referencia, diatomita natural, diatomita calcinada y diatomita calcinada con fundente. El cemento de referencia se obtuvo en base a dosificaciones de diversas bloqueras y se llama R. Los bloques de pumicita mezclados con diatomita natural se obtuvieron reemplazando el cemento con diatomita natural en varias proporciones $(5 \%, 10 \%$ 
y $15 \%$ ) en peso y manteniendo la pumicita como se aprecia claramente en la Tabla 1. Estas muestras fueron llamadas 5DN, $10 \mathrm{DN}$ y $15 \mathrm{DN}$, respectivamente. De manera similar, las muestras de block mezclado con diatomita calcinada se obtuvieron reemplazando el cemento con diatomita calcinada en varias proporciones $(5 \%, 10 \%$ y $15 \%)$ en peso y manteniendo la pumicita (Tabla 1). Estas muestras fueron llamadas 5DC, 10DC y 15DC, respectivamente. Por último, las muestras de block mezclado con diatomita calcinada con fundente se obtuvieron reemplazando el cemento con diatomita calcinada con fundente en varias proporciones $(5 \%, 10 \%$ y $15 \%)$ en peso y manteniendo la pumicita (Tabla 1). Estas muestras fueron llamadas 5DCF, 10DCF y 15DCF, respectivamente. Los bloques utilizados para las segundas pruebas se obtuvieron mezclando la pumicita con los agregados reciclados en porcentajes de 10,20 y $30 \%$, manteniendo constante la cantidad de cemento y diatomita.

Tabla 1. Diseño de composición del bloque de pumicita con distintos tipos de diatomita.

\begin{tabular}{cccccc}
\hline $\begin{array}{c}\text { Tipos de } \\
\text { diatomita }\end{array}$ & $\begin{array}{c}\text { Reemplazo de } \\
\text { Diatomita }(\%)\end{array}$ & $\begin{array}{c}\text { Diatomita } \\
(\mathrm{g})\end{array}$ & $\begin{array}{c}\text { Cemento } \\
(\mathrm{g})\end{array}$ & $\begin{array}{c}\text { Pumicita } \\
(\mathrm{g})\end{array}$ & $\begin{array}{c}\text { Agua } \\
(\mathrm{g})\end{array}$ \\
\hline Diatomita & 5 & 5.4 & 102 & & \\
Calcinada con & 10 & 10.8 & 96.5 & 964.3 & 116 \\
fundente & 15 & 16.2 & 91 & & \\
$(\mathrm{NaOH})(\mathrm{DCF})$ & & 5.4 & 102 & & \\
Diatomita & 5 & 10.8 & 96.5 & 964.3 & 116 \\
Calcinada sin & 10 & 16.2 & 91 & & \\
fundente (DC) & 15 & 5.4 & 102 & & \\
Diatomita & 5 & 10.8 & 96.5 & 964.3 & 116 \\
Natural (DN) & 10 & 16.2 & 91 & & \\
\hline
\end{tabular}

Para las pruebas de resistencia a la compresión, se realizaron bloques de pumicita con un tamaño de 60 x 200 x $50 \mathrm{~mm}$. Se colocaba la mezcla y se comprimía con un pisón, una vez enrasados y comprimidos, se desmoldaba y se dejaban secar a temperatura ambiente durante 7, 14 y 28 días para realizarle las pruebas de resistencia a la compresión. Las pruebas se realizaron en base a la normativa norma NOMNMX-C-036 utilizando una prensa ELVEC

\section{RESULTADOS Y DISCUSIÓN}

\subsection{Análisis químico}

En las tablas 2, 3 y 4 se presentan los resultados de XRF. Cabe mencionar que solo se muestran los principales óxidos, porque el propósito es conocer la variación del calcio en la pasta de cemento y la cantidad de óxido de silicio en las diatomitas.

Tabla 2. Análisis de Fluorescencia de Rayos X de Agregados Reciclados.

\begin{tabular}{ccccccc}
\hline Óxidos & $\mathrm{SiO} 2$ & $\mathrm{CaO}$ & $\mathrm{Al} 2 \mathrm{O} 3$ & $\mathrm{Fe} 2 \mathrm{O} 3$ & $\mathrm{~K} 2 \mathrm{O}$ & $\mathrm{TiO} 2$ \\
\hline Agregados reciclados & 48.368 & 26.526 & 8.423 & 4.572 & 1.118 & 0.516 \\
\hline
\end{tabular}

Tabla 3. Análisis de Fluorescencia de Rayos X de Diatomita.

\begin{tabular}{ccccccc}
\hline Óxidos & $\mathrm{SiO} 2$ & $\mathrm{Al} 2 \mathrm{O} 3$ & $\mathrm{Fe} 2 \mathrm{O} 3$ & $\mathrm{MgO}$ & $\mathrm{K} 2 \mathrm{O}$ & $\mathrm{CaO}$ \\
\hline Diatomita & 77.135 & 7.77 & 2.344 & 1.869 & 1.115 & 1.041 \\
\hline
\end{tabular}


Tabla 4. Análisis de Fluorescencia de Rayos X de Pumicita.

\begin{tabular}{ccccccc}
\hline Óxidos & $\mathrm{SiO} 2$ & $\mathrm{~A} 12 \mathrm{O} 3$ & $\mathrm{~K} 2 \mathrm{O}$ & $\mathrm{Fe} 2 \mathrm{O} 3$ & $\mathrm{CaO}$ & $\mathrm{TiO} 2$ \\
\hline Pumicita & 72.983 & 15.28 & 5.00 & 3.176 & 1.378 & 0.298 \\
\hline
\end{tabular}

\subsection{Composición mineralógica}

En los diagramas de DRX de las materias primas (Fig. 2), se observó una extensa estructura amorfa en la pumicita y diatomita. Se considera que la pumicita es ácida y los agregados del concreto reciclado y la diatomita de composición intermedia. Los feldespatos son triclínicos y pueden ser albita, anortita, andesina y oligoclasa, en pocas palabras son plagioclasas y por su parte el piroxeno parece ser enstatita. (tabla 5).

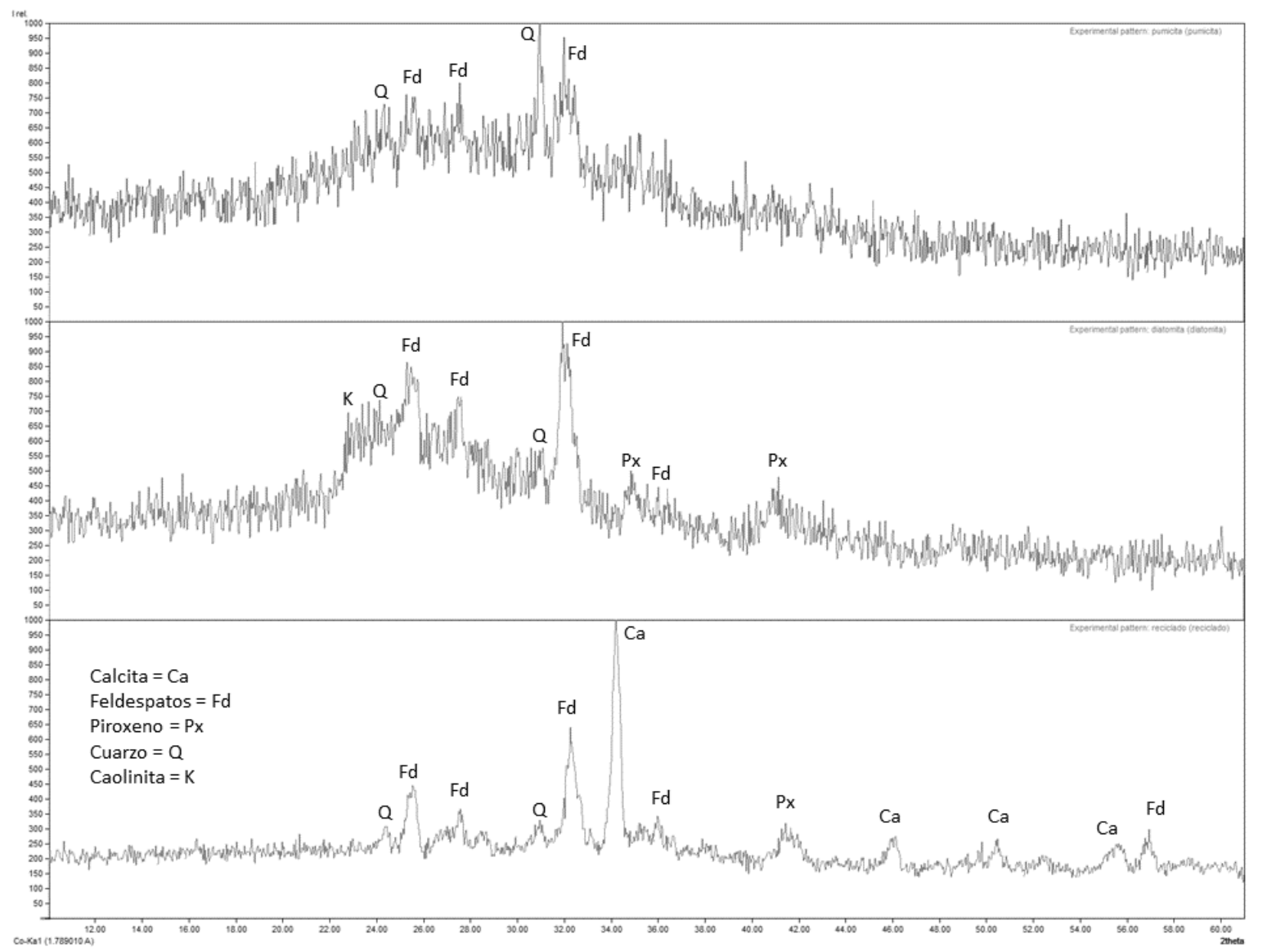

Figura 2. Espectros de DRX donde se aprecian las principales fases mineralógicas presentes. Los espectros son: a) Pumicita b) Diatomita y c) Agregados Reciclados

Tabla 5. Fases mineralógicas presentes en las materias primas.

\begin{tabular}{cccc}
\hline & Pumicita & Diatomita & $\begin{array}{c}\text { Agregados } \\
\text { Reciclados }\end{array}$ \\
\hline Feldespatos & $* *$ & $* *$ & $* * *$ \\
Calcita & - & - & $* *$ \\
Cuarzo & $* * *$ & $* *$ & $*$ \\
Piroxeno & - & $*$ & $*$ \\
Caolinita & - & $*$ & - \\
\hline & Proporciones $* * *$ Mayor $* *$ Mediana $*$ Menor
\end{tabular}




\subsection{Análisis Microestructural}

La estructura microscópica de las muestras de las materias primas se examinó utilizando las imágenes de MEB. Se observó que las diatomitas naturales (Figura 3) y calcinadas (figura 4) tenían formas similares, pero de diferente tamaños. Se observaron variaciones en la estructura de la diatomita natural con respecto a la diatomita calcinada con formas irregulares dominantes en las segundas. En las micrografías de los agregados reciclados (Figura 5) se aprecian algunos feldespatos y silicatos, con presencia de calcita cubriendo algunas partes. En la pumicita (Figura 6) se aprecian partículas con bordes afilados, lo que es representativo de silicatos y poros característicos de las espumas volcánicas.

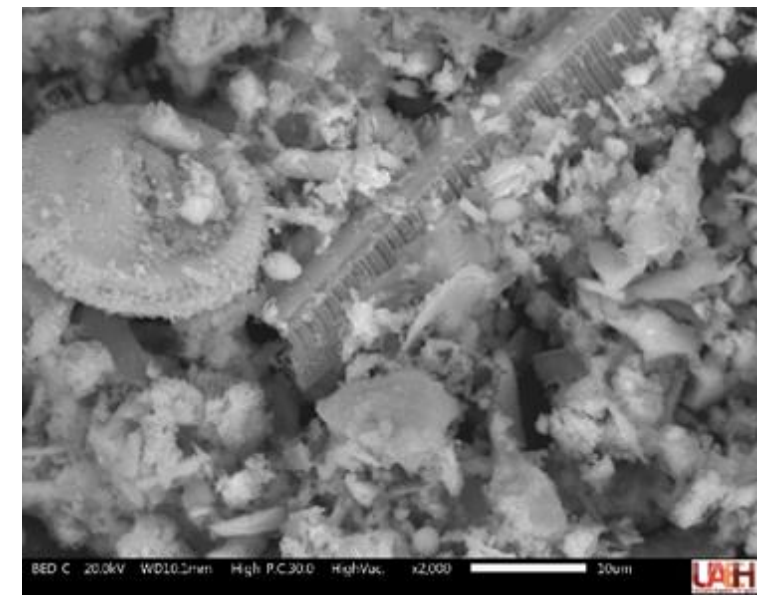

Figura 3. Diatomita natural

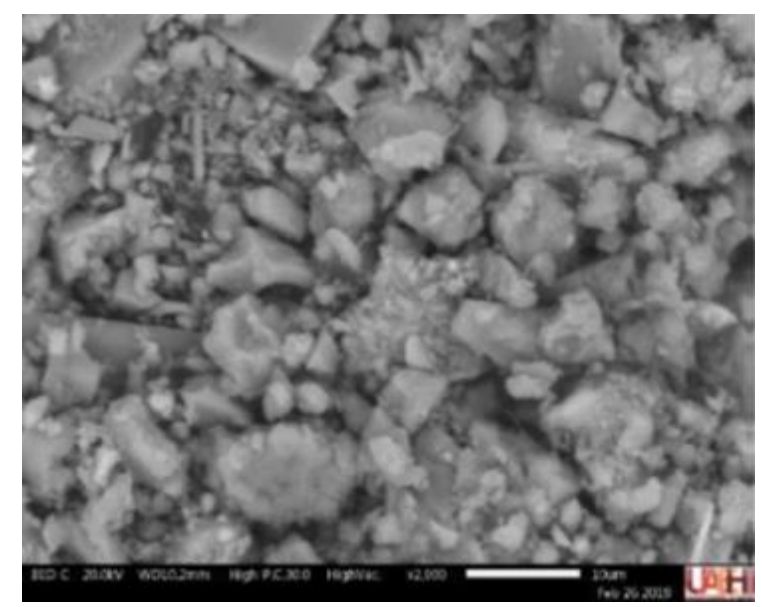

Figura 5. Agregados reciclados

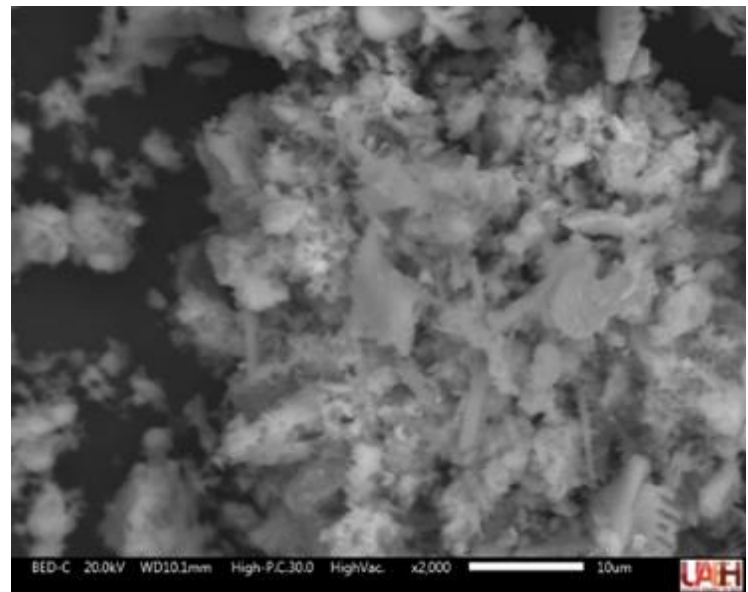

Figura 4. Diatomita calcinada

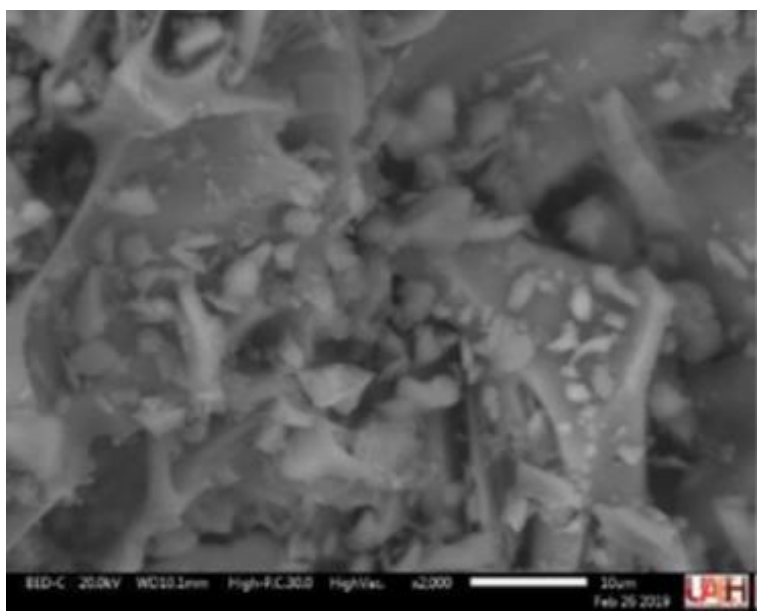

Figura 6. Pumicita

Los datos del microanálisis por energías dispersas (EDS) (tablas 6) muestran la proporción semicuantitativa elemental en peso de la pumicita, donde el silicio y aluminio prevalecen siendo característicos de los vidrios volcánicos. Así mismo, el microanálisis EDS (tablas 7,8,9) de las diatomitas, se aprecia principalmente el silicio, consistente con la intensidad de los picos del dióxido de silicio en DRX. Por último, los resultados del microanálisis EDS de los agregados reciclados (tabla 10) nos muestran calcio en su mayoría, convergiendo con los datos obtenidos en la composición mineralógica por difracción de Rayos X, donde la fase principal fue la calcita. 
Tabla 6. Análisis semicuantitativo de elementos presentes en la muestra de Pumicita.

\begin{tabular}{cc}
\hline Elemento & \% en peso \\
\hline $\mathrm{O}$ & 48.68 \\
$\mathrm{Na}$ & 2.28 \\
$\mathrm{Mg}$ & 0.59 \\
$\mathrm{Al}$ & 7.18 \\
$\mathrm{Si}$ & 34.79 \\
$\mathrm{~K}$ & 4.77 \\
$\mathrm{Fe}$ & 1.71 \\
Total: & 100.00 \\
\hline
\end{tabular}

Tabla 7. Análisis semicuantitativo de elementos presentes en la muestra de Diatomita Natural.

\begin{tabular}{cc}
\hline Elemento & \% en peso \\
\hline $\mathrm{C}$ & 20.16 \\
$\mathrm{O}$ & 67.37 \\
$\mathrm{Mg}$ & 0.20 \\
$\mathrm{Al}$ & 0.71 \\
$\mathrm{Si}$ & 11.23 \\
$\mathrm{Ca}$ & 0.13 \\
$\mathrm{Fe}$ & 0.22 \\
Total: & 100.00 \\
\hline
\end{tabular}

Tabla 8. Análisis semicuantitativo de elementos presentes en la muestra de Diatomita Calcinada.

\begin{tabular}{cc}
\hline Elemento & \% en peso \\
\hline $\mathrm{O}$ & 52.23 \\
$\mathrm{Al}$ & 2.31 \\
$\mathrm{Si}$ & 43.60 \\
$\mathrm{~K}$ & 0.75 \\
$\mathrm{Ca}$ & 0.30 \\
$\mathrm{Fe}$ & 0.80 \\
Total: & 100.00 \\
\hline
\end{tabular}

Tabla 9. Análisis semicuantitativo de elementos presentes en la muestra de Diatomita Calcinada con Fundente.

\begin{tabular}{cc}
\hline Elemento & \% en peso \\
\hline $\mathrm{O}$ & 50.87 \\
$\mathrm{Na}$ & 1.06 \\
$\mathrm{Mg}$ & 0.88 \\
$\mathrm{Al}$ & 3.62 \\
$\mathrm{Si}$ & 40.09 \\
$\mathrm{~K}$ & 0.65 \\
$\mathrm{Ca}$ & 0.70 \\
$\mathrm{Fe}$ & 2.14 \\
Total: & 100.00 \\
\hline
\end{tabular}


Tabla 10. Análisis semicuantitativo de elementos presentes en la muestra de Agregado Reciclado.

\begin{tabular}{cc}
\hline Elemento & \% en peso \\
\hline $\mathrm{O}$ & 39.35 \\
$\mathrm{Mg}$ & 0.45 \\
$\mathrm{Al}$ & 3.95 \\
$\mathrm{Si}$ & 18.73 \\
$\mathrm{~K}$ & 1.06 \\
$\mathrm{Ca}$ & 31.39 \\
$\mathrm{Fe}$ & 5.07 \\
Total: & 100.00 \\
\hline
\end{tabular}

\subsection{Resistencia a la compresión}

La influencia de los tipos de diatomitas en la resistencia del cemento Portland se aprecia en el gráfico de la figura 7 donde se observa la resistencia a la compresión de la muestra del bloque de pumicita con cemento Portland mezclado con diferentes tipos y contenidos de diatomita a la temperatura de curado de $20^{\circ} \mathrm{C}$ a $\operatorname{los} 7,14$ y 28 días.
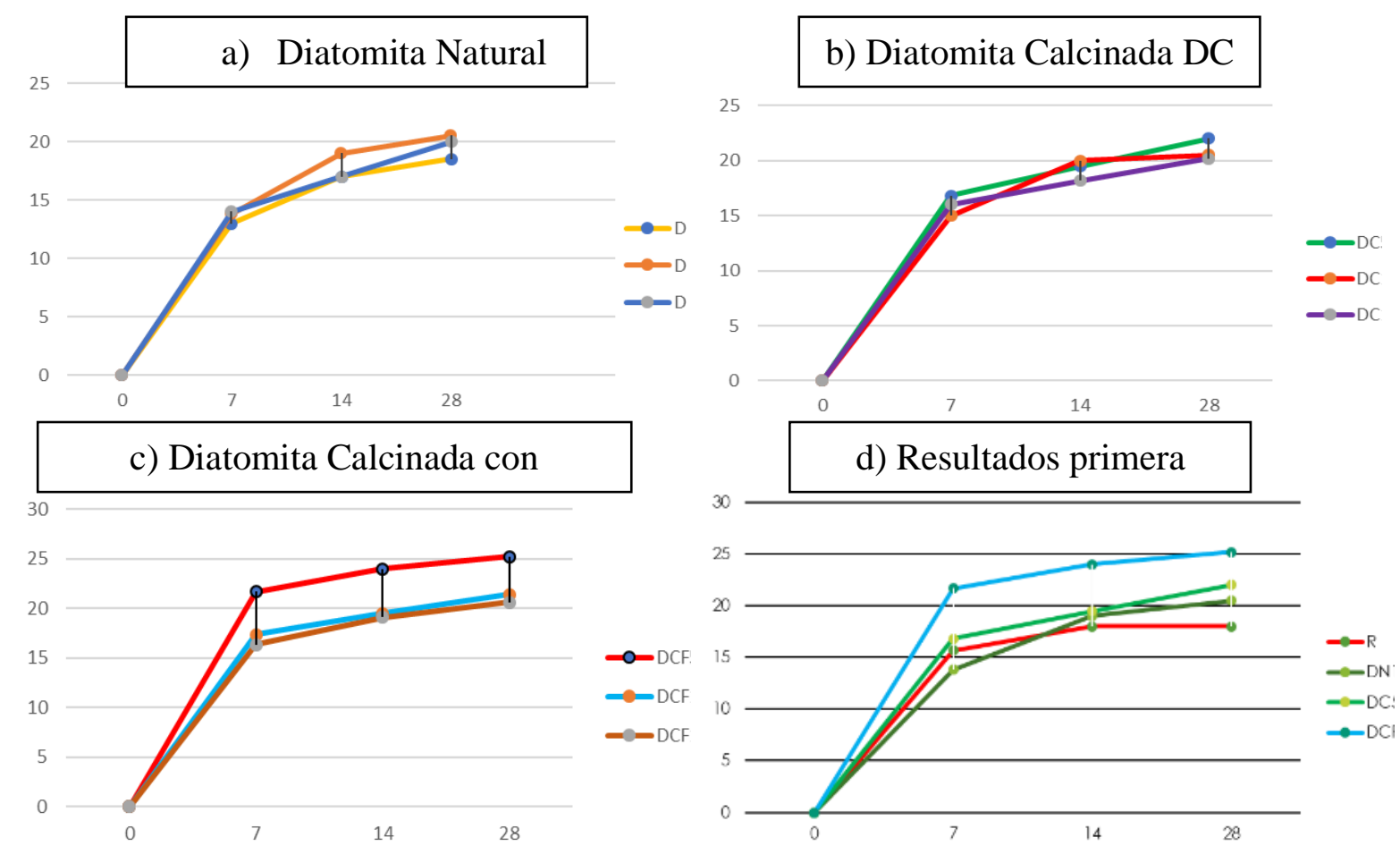

Figura 7. La resistencia a la compresión del bloque de pumicita mezclado con diferentes tipos de diatomita (a)

La muestra mezclada con diatomita normal DN (b) La muestra mezclada con diatomita calcinada DC (c) La muestra mezclada con diatomita calcinada con fundente DCF d) Comparativa de los resultados óptimos obtenidos de cada prueba contra el Block normal.

Resultando que la resistencia de la muestra de block con diferentes contenidos de diatomita fue ligeramente inferior en la Diatomita Natural a la de la muestra de Referencia; a la edad de 7 días, las resistencias de la muestra de block de pumicita mezclado con la cantidad de mezcla de Diatomita Calcinada (DC) y Diatomita Calcinada con Fundente (DCF) fueron en su mayoría más altas que la de la muestra de Referencia (R). 
En promedio las resistencias con la cantidad de mezcla del $5 \%$ en sustitución con el cemento de DC y DCF son $22 \mathrm{~kg} / \mathrm{cm} 2$ y $24.4 \mathrm{~kg} / \mathrm{cm} 2$ respectivamente siendo superiores a las de las muestras con la cantidad de mezcla del $10 \%$ y el $15 \%$. Se comparan con la resistencia del block normal $(20 \mathrm{~kg} / \mathrm{cm} 2)$ contra las muestras de DC y DCF, aumentando entre un $10 \%$ y $22 \%$ respectivamente. Dando como resultado que la Diatomita Calcinada con Fundente mezclada con el cemento a un 5\% puede mejorar la resistencia del espécimen de block de pumicita a temprana edad ( 7 días) en un $22 \%$ con respecto al block convencional.

El Ca $(\mathrm{OH})_{2}$ (Hidróxido de Calcio) se produjo por la hidratación del $\mathrm{SiO}_{2}$ (dióxido de Silicio) y las partículas de cemento en las partículas de diatomita y el gel de C-S-H (Silicatos de Calcio Hidratados) generado por $\mathrm{SiO}_{2}$ en la reacción de hidratación secundaria para aumentar la resistencia a la compresión del cemento (Liu et al, 2016).

Posteriormente la influencia del Agregado reciclado en la resistencia del bloque de pumicita se aprecia en el gráfico de la figura 8 a la temperatura de curado de $20^{\circ} \mathrm{C}$ a $\operatorname{los} 7,14$ y 28 días.

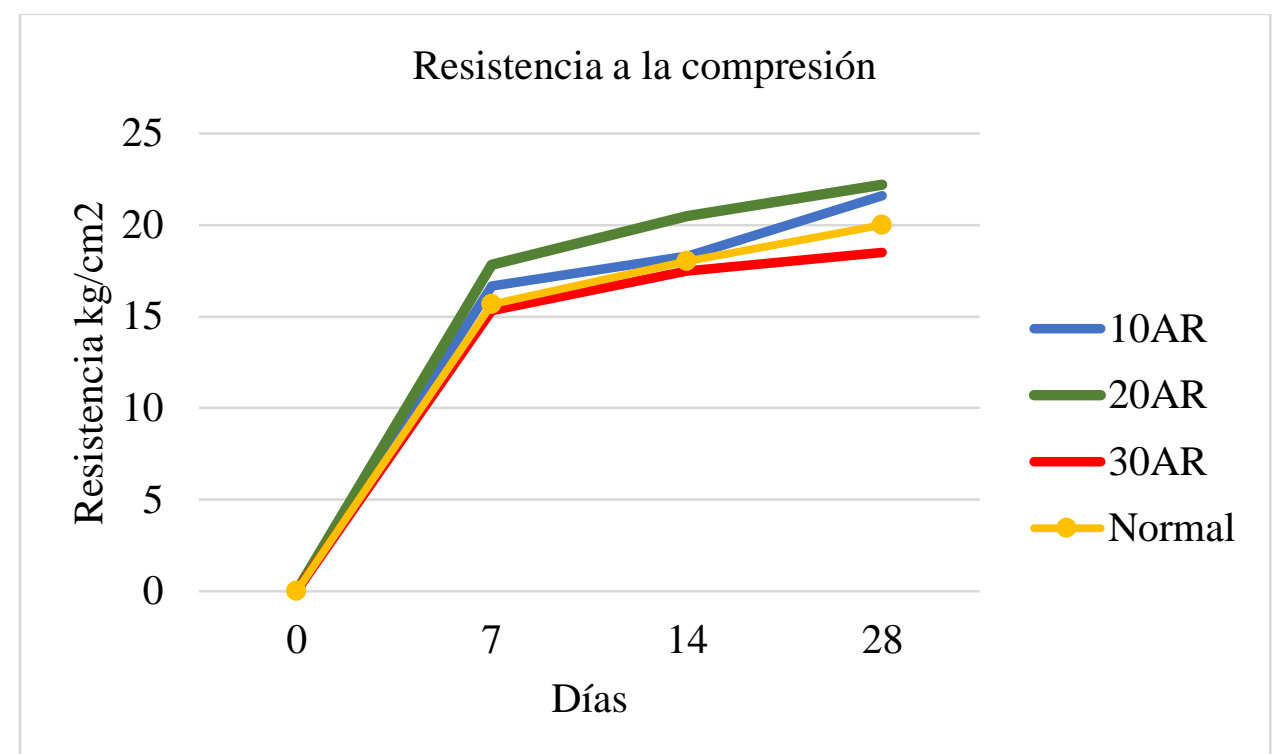

Figura 8. Gráfico que muestra la resistencia a la compresión de las muestras de bloques con agregados reciclados al 10\% (10AR); agregados reciclados al 20\% (20AR); agregados reciclados al 30\% (30AR), a los 7, 14 y 28 días.

En la figura 8 se aprecia que 2 muestras de bloques de pumicita con agregados reciclados 10AR y 20AR son mayores, $21.6 \mathrm{~kg} / \mathrm{cm} 2$ y $22.2 \mathrm{~kg} / \mathrm{cm}^{2}$ respectivamente, que las del bloque de referencia $\left(20 \mathrm{~kg} / \mathrm{cm}^{2}\right)$, el que contiene el $30 \%$ de agregados reciclados en sustitución de la pumicita resultó menos eficiente $\left(18.5 \mathrm{~kg} / \mathrm{cm}^{2}\right)$; En base a los resultados se elige el bloque 20AR cuya resistencia aumentó en un $12 \%$ con respecto al bloque de referencia. Es importante mencionar que la resistencia alcanzada es ligeramente menor que la obtenida anteriormente solo con la sustitución de la diatomita calcinada con fundente (10\% menor).

La calcita $\left(\mathrm{CaCO}_{3}\right)$ aparece en proporciones menores en todos los agregados reciclados. Por lo tanto, la calcita se debe predominantemente a la carbonatación de los productos de la hidratación del cemento en la pasta adherida a los agregados reciclados, como la portlandita $\mathrm{Ca}(\mathrm{OH})_{2}$ y los silicatos de calcio hidratado C-S-H $\left(\mathrm{CaO}-\mathrm{SiO}_{2}-\mathrm{H}_{2} \mathrm{O}\right)$. El carbonato de calcio se considera un cementante débil (Moreno et al, 2018), por eso disminuye su resistencia. Estas fases están carbonatadas como se describe en las Reacciones (1) y (2) (Shi et al, 2016): 


$$
\begin{aligned}
& \mathrm{Ca}(\mathrm{OH})_{2}+\mathrm{CO}_{2} \rightarrow \mathrm{CaCO}_{3}+\mathrm{H} 2 \mathrm{O} \\
& \mathrm{C}-\mathrm{S}-\mathrm{H}+\mathrm{CO} 2 \rightarrow \mathrm{CaCO} 3+\mathrm{SiO} 2 \cdot \mathrm{nH} 2 \mathrm{O}
\end{aligned}
$$

Se determinó que el bloque elaborado con un $20 \%$ de agregado reciclado es el óptimo debido a que presenta una resistencia a la compresión de $22.2 \mathrm{~kg} / \mathrm{cm}^{2}$ mayor al de block de referencia $20 \mathrm{~kg} / \mathrm{cm}^{2} \mathrm{y}$ ligeramente superior al de los otros bloques con el $10 \%$ y $30 \%$ de agregado reciclado (21.6 y 18.5 $\mathrm{kg} / \mathrm{cm}^{2}$ respectivamente). Se procede a realizar la técnica de caracterización de Microscopía Electrónica de Barrido para hacer una comparativa entre el block de pumicita comercial común y el block con agregados reciclados y diatomita; obteniendo una imagen microestructural donde se encuentren las formaciones de silicatos cálcicos hidratados $\mathrm{C}-\mathrm{S}-\mathrm{H}$, cuya función es la de darle resistencia al cemento.

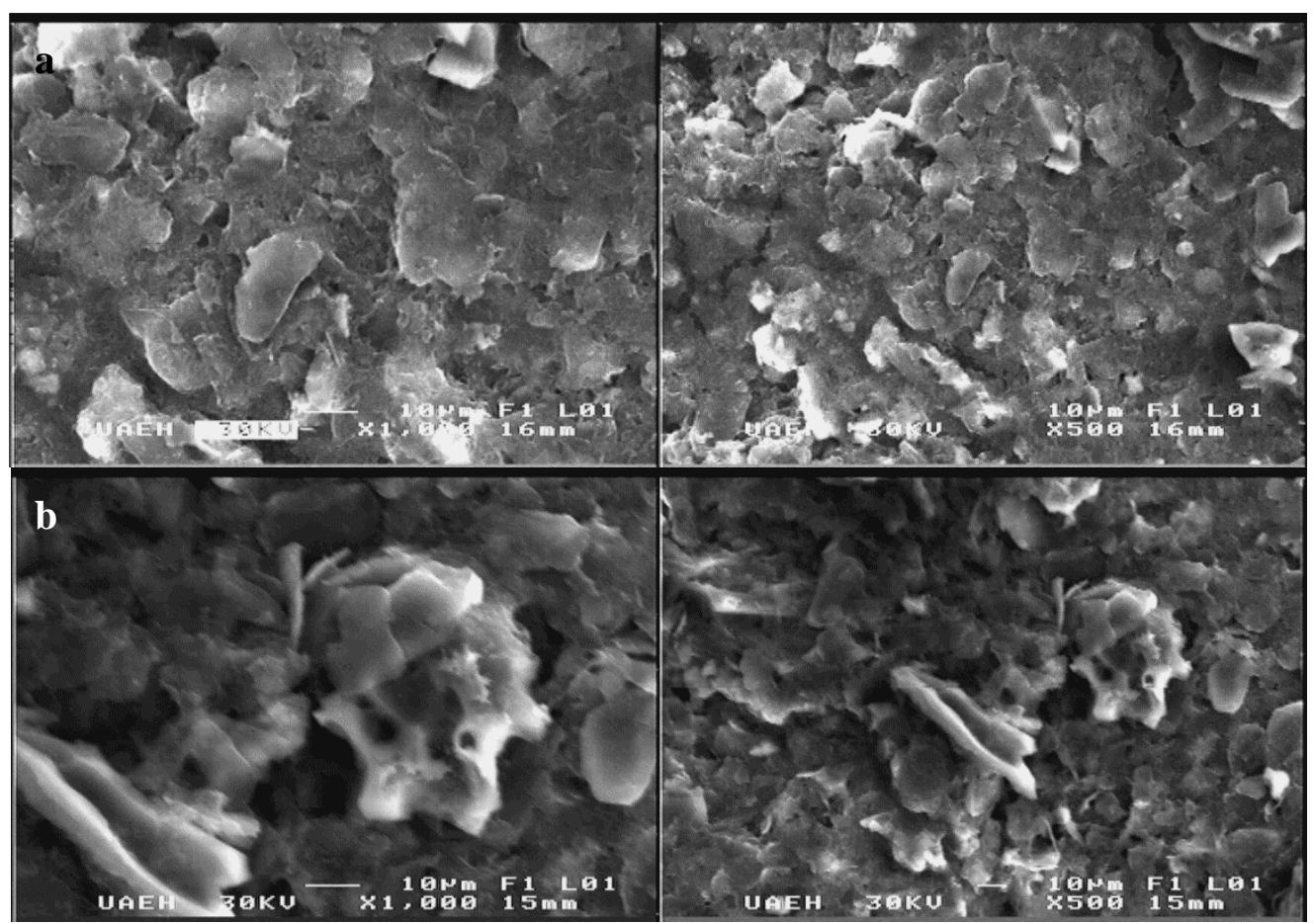

Figura 9. Micrografías donde se puede cotejar la presencia de C-S-H en un a) bloque común y un b) bloque con diatomita al 5\% y Agregados Reciclados al $20 \%$.

En la figura 9 se puede observar la fracción de arena de cuarzo y la porosidad esférica producto de aditivos aireantes junto con la cantidad de C-S-H que es mayor en el bloque b) lo que conlleva a coadyuvar el aumento de la resistencia del bloque y respaldan los resultados obtenidos en las pruebas de resistencia mecánica de la figura 8 . Se aprecia que la diatomita está reaccionando con el cemento generando los silicatos de calcio hidratados, por lo que se nota mayor presencia de ellos cerca de ésta. En la figura 10 se nota la forma de un gel de C-S-H cuya longitud es de aproximadamente 10 micras y su forma de aguja está definida. 


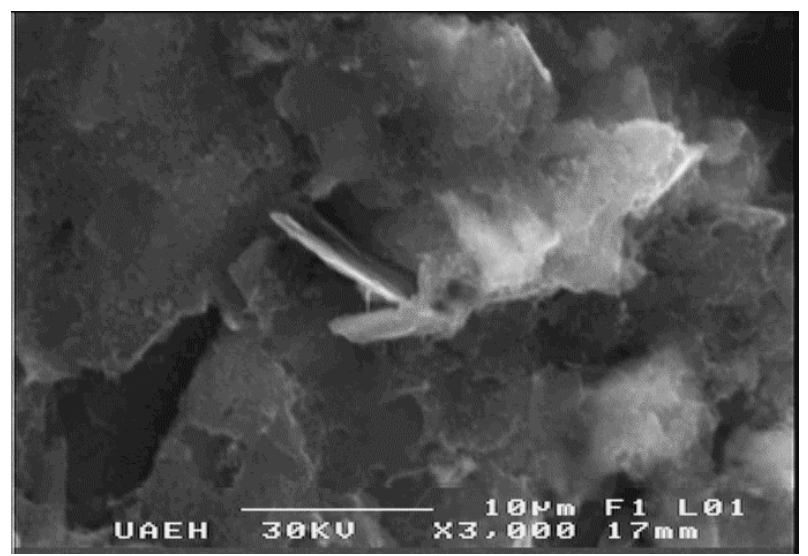

Figura 10. Micrografía donde se aprecia un gel de C-S-H a 3,000 aumentos y un voltaje de $30 \mathrm{KV}$.

\section{CONCLUSIONES}

En la primera etapa de elaboración de bloques de pumicita, se observó un incremento en la resistencia a la compresión a temprana edad (7 días) con la sustitución del 5\% de diatomita calcinada con fundente (DCF). Incrementando un $22 \%$ a una edad de 28 días, que es cuando el concreto alcanza una resistencia cercana al 99\%. Notándose que a una edad de 7 días presenta un aumento del $20 \%$ comparado a un bloque de pumicita convencional. Esto ocurre debido a que el $\mathrm{Ca}(\mathrm{OH})_{2}$ (Hidróxido de Calcio) se produjo por la hidratación del $\mathrm{SiO}_{2}$ (dióxido de Silicio) y las partículas de cemento en las partículas de diatomita y el gel de CSH (Silicatos de Calcio Hidratados) generado por $\mathrm{SiO}_{2}$ en la reacción de hidratación secundaria para aumentar la resistencia a la compresión del cemento. Al aumentar la cantidad de diatomita se producen más partículas de ésta que al no reaccionar sólo perjudican la resistencia de la pasta de cemento (Liu et al, 2016), por lo cual se recomienda dejar fraguar hasta los 28 días.

La dosificación de los bloques de pumicita para la segunda etapa de elaboración, donde se sustituye el agregado reciclado por la pumicita, se llevó a cabo en base a los pesos específicos de los 2 agregados, resultando un ligero aumento de peso de $45 \mathrm{~g}$ en el bloque con $20 \%$ de Agregado Reciclado y un aumento de la cantidad de agua de un $7 \%$.

El bloque con el $20 \%$ de Agregado reciclado aumentó la resistencia del block de pumicita normal en un 12\%, no mostrando aumento a edad temprana, sino hasta los 14-28 días de curado. Disminuyendo un $10 \%$ comparado con la primera etapa de elaboración de bloques. Esto ocurre por la carbonatación de los productos de la hidratación del cemento en la pasta adherida a los agregados reciclados, como la portlandita $\mathrm{Ca}(\mathrm{OH})_{2}$ y los silicatos de calcio hidratado $\mathrm{C}-\mathrm{S}-\mathrm{H}\left(\mathrm{CaO}-\mathrm{SiO}_{2}-\mathrm{H}_{2} \mathrm{O}\right)$ como lo menciona Moreno et al, 2018, reduciendo la resistencia del producto.

Si bien el aumento con el árido reciclado fue menor que el obtenido sólo con la diatomita, sigue siendo mayor en comparación con el bloque de pumicita convencional. Haciendo énfasis en la parte sustentable del estudio, ya que se está reduciendo el impacto ambiental generado por los Residuos de Construcción y Demolición, proporcionalmente a la disminución de la pumicita al incorporarlo al block.

Siguiendo en el ámbito ambiental, al sustituir la diatomita por cemento, se está disminuyendo la huella de carbono generada por el cemento portland compuesto en su elaboración, haciendo cuentas por cada tonelada de cemento ( 20 bultos) utilizada en la elaboración de blocks de pumicita, se estaría ahorrando $50 \mathrm{~kg}$ de cemento (1 bulto). Según la revista Civilizate, No. 8, 2016 el cemento convencional produce en promedio 0,8 toneladas de $\mathrm{CO}_{2}$ por cada tonelada de cemento; con lo que se estaría reduciendo 40 $\mathrm{kg}$ de $\mathrm{CO}_{2}$ por tonelada de cemento producida. En base a Barker et al, 2009. La industria del cemento contribuye con alrededor del $7 \%$ del total de las emisiones mundiales de $\mathrm{CO}_{2}$ (aproximadamente $1.8 \mathrm{Gt}$ de emisiones de $\mathrm{CO}_{2}$ al año). Por lo tanto, se reduciría unas .09 Gt de emisiones de $\mathrm{CO}_{2}$ al año. 
En base a datos obtenidos por diversas casas de materiales, con un bulto de cemento se pueden fabricar de 30 a 35 bloques de pumicita, en base al uso del 20\% de agregados reciclados en sustitución con la pumicita, se estaría ahorrando de 7 a 8 bloques de pumicita por cada bulto de cemento, usándose para elaborar otros bloques.

\section{REFERENCIAS}

Akhtar, A., Sarmah, A. (2018). Construction and demolition waste generation and properties of recycled aggregate concrete: A global perspective. Journal of Cleaner Production, 10.1016, p.6.

Aydin, A. C., Gül, R. (2007). Influence of volcanic originated natural materials as additive on the setting time and some mechanical properties of concrete. Construction and building materials, 21:1277-81.

Borgel, I. (2007). Caracterización del yacimiento de diatomita de Loma Larga, Municipio de Acatlán, Hidalgo y evaluación de sus aplicaciones alternas. Tesis de Licenciatura, Área Académica de Materiales y Metalurgia, Universidad Autónoma del Estrado de Hidalgo.

Kastis, D. (2006), Properties and hydration of blended cements with calcareous diatomite. Cement Concrete Research, 36:1821-6.

Liu, J., Shao, P., Wang, S. (2016). The Influence of Diatomite on the Strength and Microstructure of Portland Cement. MATEC Web of Conferences, 67, 07-17.

Mete Z. Benefication of Kütahya-Alayunt Diatomite Ores (in Turkish). Akdeniz University Engineering Faculty Journal, Mining Section 1988;1:184-201.

Moreno, E., Hernández, J., Rangel, Y., Cerecedo, E., Arenas, A., Reyes, M., \& Salinas, E. (2018). Chemical and mineralogical characterization of recycled aggregates from construction and demolition waste from Mexico City. Minerals, 8, p. 237.

Oh, D.Y., Noguchi, T., Kitagaki, R., Park, W. J. (2014), CO2 emission reduction by reuse of 14 building material waste in the Japanese cement industry. Renew. Sustain. Energy Rev. 15 38, 796-810. https://doi.org/10.1016/j.rser.2014.07.036

Serralde, R., Sánchez, D., y López, J. (2015). Factibilidad de implementación de una empresa de base tecnológica para la fabricación de elementos urbanos, elaborados con base en residuos de la construcción y demolición (RCD): Analisis técnico de productos y maquinaria. Tesis de Licenciatura, Área Académica de Ingeniería, Universidad Autónoma del Estrado de Hidalgo.

Shi, C.; Li, Y.; Zhang, J.; Li, W. (2016), Chong, L.; Xie, Z. Performance enhancement of recycled aggregate-A review. J. Clean. Prod. 112, 466-472.

Stamatakis, M. G., Fragoulis, D., Csirik, G., Bedelean, I., Pedersen, S. (2003), The influence of biogenic micro-silica-rich rocks on the properties of blended cements. Cement Concrete Composites. 25:17784. 